03

\title{
О дифракционных явлениях в сенсорах на поверхностных волнах
}

\author{
(C) А.Б. Петрин \\ Объединенный институт высоких температур РАН, \\ 125412 Москва, Россия \\ e-mail: a_petrin@mail.ru
}

Поступила в редакцию 15.05.2018 г.

На основе теоретического метода исследования отражения плоской электромагнитной волны от плоскослоистой многопленочной структуры с однородными пленками рассмотрены дифракционные явления, связанные с ограниченным размером апертуры падающей волны. Предложенная теория применяется к анализу чувствительности сенсоров на поверхностных волнах.

DOI: $10.21883 /$ OS.2018.09.46554.126-18

\section{Введение и постановка задачи}

В настоящее время большое внимание уделяется созданию многоканальных оптических сенсорных систем, применяемых в биологических, химических и физических исследованиях и использующих поверхностные плазмонные (или плазмонно-поляритонные) волны для диагностики изменений распространения поверхностной волны, вызванных изменением поверхностных свойств исследуемой среды [1-4]. Такие волны, например, распространяются вдоль поверхности металла, локализуясь вблизи его поверхности [5,6]. Часто используемым способом возбуждения поверхностных плазмонов является метод Кречмана [7].

В рамках схемы Кречмана [8] (рис. 1) согласование поверхностной плазмонной волны 1 на поверхности металлической пленки 2 и падающей волны 3 осуществляется с помощью стеклянной призмы 4. Линейно поляризованная монохроматическая волна $\mathbf{E}^{i}$ падает на металлическую пленку со стороны призмы. Вектор электрического поля падающей волны направлен в плоскости падения ( $p$-поляризация). Призма необходима для согласования падающей плоской волны и поверхностной плазмонной волны по волновому числу. Волновое число поверхностной плазмонной волны больше волнового числа плоской волны в свободном пространстве той же частоты $[9,10]$. Чтобы обеспечить равенство волновых чисел падающей 3 , отраженной 5 и поверхностной плазмонной 1 волн вдоль поверхности металла (рис. 1) необходимо, чтобы падающая волна подходила к пленке из оптически более плотной среды (где волновое число больше, чем в свободном пространстве). Показатель преломления призмы и угол падения подбираются таким образом, чтобы обеспечивалось равенство волновых чисел падающей и поверхностных волн. При этих параметрах в отсутствие металлической пленки (и возбуждения поверхностных плазмонов) наблюдается полное внутреннее отражение падающей волны.
Эксперименты показывают, что, если угол падения соответствует согласованию по волновому числу падающей и поверхностной плазмонной волн, наблюдается резкое падение коэффициента отражения. Например, при длине падающей волны в вакууме $\lambda=633 \mathrm{~nm}$ и толщине пленки серебра приблизительно равной $53.8 \mathrm{~nm}$ (эта величина зависит от конкретного значения показателя преломления призмы и частоты падающей волны) наблюдается сильное изменение коэффициента отражения практически от единицы до нуля в окрестности угла падения, соответствующего согласованию свободной и поверхностной плазмонной волн. При небольшом отклонении угла падения от угла согласования коэффициент отражения возвращается практически от нуля к единичному значению.

Так как поле поверхностной плазмонной волны сосредоточено в тонком слое вблизи поверхности металла, характер отражения в схеме Кречмана сильно зависит от изменений показателя преломления в тонком слое вблизи поверхности. Именно на этом основывается широкое применение схемы Кречмана для создания различного рода датчиков, обладающих высокой чувствительностью [11]. Исследования пока-

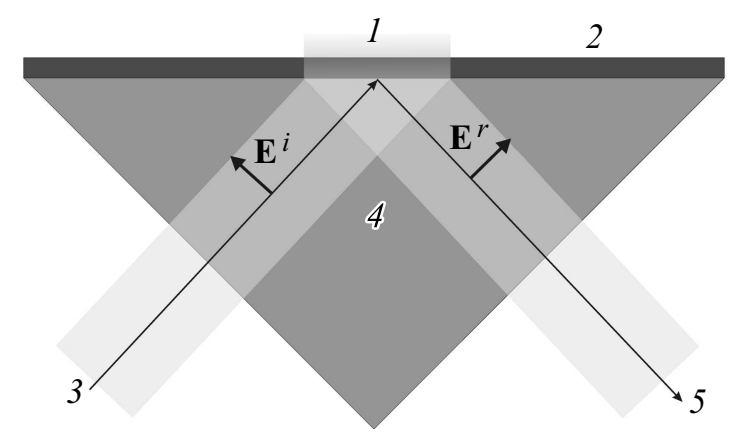

Рис. 1. Возбуждение поверхностных плазмонов 1 на поверхности металлической пленки 2 по схеме Кречмана. Падающая волна 3 в призме 4 порождает поверхностную плазмонную волну 1 и отраженную волну 5 . 
зали, что чувствительность определяется угловой шириной минимума отражения [1], которая, в свою очередь, определяется поглощением в металле пленки. Чем меньше поглощение в металле, тем уже минимум.

Чтобы увеличить чувствительность датчиков за счет уменьшения угловой ширины минимума, было предложено уменьшать толщину пленки металла. Оказалось, что у поверхностной плазмонной волны в тонкой пленке затухание уменьшается с уменьшением толщины пленки. Однако для согласования поверхностной волны в схеме Кречмана потребовалось введение дополнительного согласующего слоя между призмой и металлической пленкой [13-16], причем в некоторых случаях даже многопленочного слоя $[17,18]$.

Отметим, что поверхностные волны могут распространяться и в диэлектрических пленках планарных волноводов [19]. В связи с этим было показано [20], что в схеме Кречмана можно возбуждать поверхностную волну (не обязательно поверхностную плазмонную), которая согласуется по волновому числу с падающей волной. В этом случае в качестве волновода поверхностной волны можно использовать пленку (или несколько пленок) оптически плотного вещества (с большим показателем преломления), отделенного от призмы пленкой согласующего вещества с низким показателем преломления. Однако в случае устройств конечных размеров (в плоскости многопленочной структуры) апертура падающей волны ограничена, поэтому отражение будет происходить не совсем так, как в случае бесконечной структуры и идеально плоских волн. В данной работе теоретически исследуется отражение ограниченных пучков и соответствующие дифракционные явления.

\section{Задача падения плоской электромагнитной волны ограниченной апертуры на плоскослоистую структуру}

Рассмотрим падение плоской монохроматической волны с циклической частотой единичной амплитуды (рис. 2) из однородного полупространства 1 (призмы), заполненного однородным изотропным диэлектриком с показателем преломления $n_{1}$, под углом $\alpha$ к нормали на плоскую пленку толщины $d_{1}$. Обобщение на произвольное количество пленок будет дано ниже. Предполагается для определенности, что пленка металлическая с комплексной диэлектрической проницаемостью $\varepsilon_{2}$ на рассматриваемой частоте $\omega$, а за пленкой находится однородное полупространство с диэлектрической проницаемостью $\varepsilon_{3}$ (свободное пространство). Пусть размер апертуры $D$ волнового фронта в плоскости $X Z$ таков, что волна падает на отрезок границы раздела длины $L$, т. е. $D=L \cos \alpha$. В дальнейшем будем пользоваться обобщениями идей работы [21, с. 377-378].

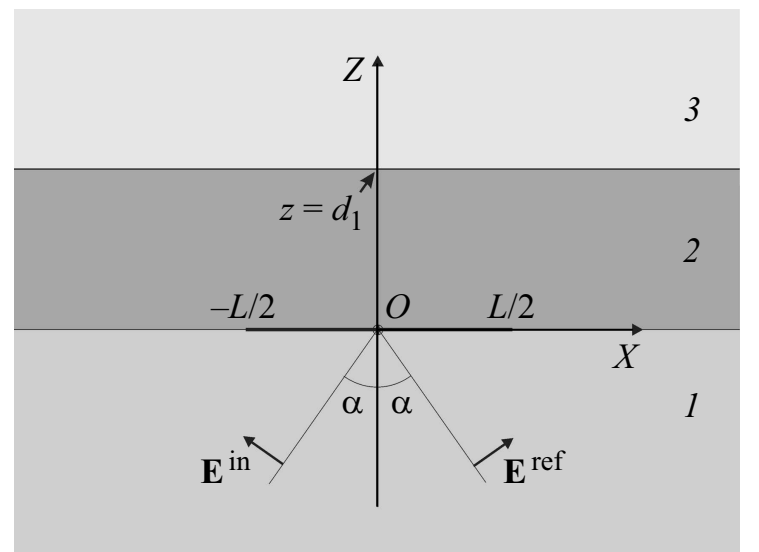

Рис. 2. Геометрия электромагнитной задачи. Плоская волна $p$ поляризации падает из призмы 1 на металлическую пленку 2, за пленкой находится вакуум 3.

Уравнения Максвелла в рассматриваемых областях можно записать в виде

$$
\begin{gathered}
\operatorname{rot} \mathbf{E}_{j}=i \omega \mathbf{B}_{j}, \\
\operatorname{rot} \mathbf{B}_{j}=-i \omega \varepsilon_{j} \mu_{j} \mathbf{E}_{j},
\end{gathered}
$$

где $\mathbf{E}_{j}$ и $\mathbf{B}_{j}$ - векторы напряженности электрического поля и индукции магнитного поля в области с номером $j ; j=1$ соответствует полупространству 1 , для которого $z<0 ; j=2$ соответствует области пленки 2 $\left(0<z<d_{1}\right) ; j=3$ соответствует свободному полупространству $\left(z>d_{1}\right)$. Комплексное представление во времени предполагается в виде $e^{-i \omega t}$.

Будем рассматривать поля, не зависящие от координаты $y$ и имеющие только $x$ - и $z$-компоненты вектора напряженности электрического поля. Из (1), (2) следует волновое уравнение для электрического поля:

$$
\operatorname{rot} \operatorname{rot} \mathbf{E}_{j}-\omega^{2} \mu_{j} \varepsilon_{j} \mathbf{E}_{j}=0
$$

Учитывая, что $\operatorname{div} \mathbf{D}_{j}=0$ или $\partial_{x} E_{j, x}+\partial_{z} E_{j, z}=0$, то

$$
\begin{aligned}
& \operatorname{rot} \operatorname{rot} \mathbf{E}_{j} \mathbf{e}_{x}\left(-\partial_{x x}^{2} E_{j, x}-\partial_{z z}^{2} E_{j, x}\right) \\
& +\mathbf{e}_{z}\left(-\partial_{z z}^{2} E_{j, z}-\partial_{x x}^{2} E_{j, z}\right),
\end{aligned}
$$

где $\mathbf{e}_{x}, \mathbf{e}_{z}$ - единичные векторы в направлении осей $X$ и $Z$.

В результате из (3) получаем уравнения для компонент $E_{j, x}$ и $E_{j, z}$ :

$$
\begin{aligned}
& \partial_{x x}^{2} E_{j, x}+\partial_{z z}^{2} E_{j, x}+\omega^{2} \mu_{j} \varepsilon_{j} E_{j, x}=0, \\
& \partial_{z z}^{2} E_{j, z}+\partial_{x x}^{2} E_{j, z}+\omega^{2} \mu_{j} \varepsilon_{j} E_{j, z}=0,
\end{aligned}
$$


Представим компоненты электрических полей в виде фурье-разложений

$$
\begin{aligned}
& E_{j, x}(x, z)=\frac{1}{2 \pi} \int_{-\infty}^{+\infty} \widetilde{E}_{j, x}(\xi, z) e^{i \xi x} d \xi, \\
& E_{j, z}(x, z)=\frac{1}{2 \pi} \int_{-\infty}^{+\infty} \widetilde{E}_{j, z}(\xi, z) e^{i \xi x} d \xi,
\end{aligned}
$$

где фурье-образы определяются интегралами

$$
\begin{aligned}
& \widetilde{E}_{j, x}(\xi, z)=\int_{-\infty}^{+\infty} E_{j, x}(x, z) e^{-i \xi x} d x \\
& \widetilde{E}_{j, z}(\xi, z)=\int_{-\infty}^{+\infty} E_{j, z}(x, z) e^{-i \xi x} d x .
\end{aligned}
$$

Тогда для фурье-образов полей из (4) и (5) получим уравнения

$$
\begin{aligned}
& \frac{d^{2} \widetilde{E}_{j, x}}{d z^{2}}+\left(\omega^{2} \mu_{j} \varepsilon_{j}-\xi^{2}\right) \widetilde{E}_{j, x}=0 \\
& \frac{d^{2} \widetilde{E}_{j, z}}{d z^{2}}+\left(\omega^{2} \mu_{j} \varepsilon_{j}-\xi^{2}\right) \widetilde{E}_{j, z}=0 .
\end{aligned}
$$

Решением этих уравнений являются $\widetilde{E}_{j, x}^{ \pm}=\hat{E}_{j, x}^{ \pm} e^{ \pm k_{j, z} z}$, $\widetilde{E}_{j, z}^{ \pm}=\hat{E}_{j, z}^{ \pm} e^{ \pm k_{j, z} z}$, где $\hat{E}_{j, x}^{+}, \hat{E}_{j, x}^{-}, \hat{E}_{j, z}^{+}, \hat{E}_{j, z}^{-}-$функции только от $\xi$, а $k_{j, z}=k_{j, z}(\xi)=\sqrt{\omega^{2} \mu_{j} \varepsilon_{j}-\xi^{2}}$.

Общее решение уравнения (3) с поляризацией в плоскости падения и заданной вариацией поля вдоль оси $X$ для направлений распространения вдоль и против оси $Z$ можно записать в виде

$$
\mathbf{E}_{j}^{ \pm}=\frac{1}{2 \pi} \int_{-\infty}^{+\infty}\left(\begin{array}{c}
\hat{E}_{j, x}^{ \pm}(\xi) \\
0 \\
\hat{E}_{j, z}^{ \pm}(\xi)
\end{array}\right) e^{ \pm i k_{j, z}(\xi) z} e^{j \xi x} d \xi
$$

где $\xi^{2}+k_{j, z}^{2}(\xi)=\omega^{2} \mu_{j} \varepsilon_{j}$. Так как $\operatorname{div} \mathbf{D}_{j}=0$, то

$$
\varepsilon_{j} \xi \hat{E}_{j, x} \pm \varepsilon_{j} k_{j, z}(\xi) \hat{E}_{j, z}=0 .
$$

Используя (9), выражение (8) можно переписать в виде

$$
\mathbf{E}_{j}^{ \pm}=\frac{1}{2 \pi} \int_{-\infty}^{\infty}\left(\begin{array}{c}
1 \\
0 \\
\mp \frac{\xi}{k_{j, z}(\xi)}
\end{array}\right) \hat{E}_{j, x}(\xi) e^{ \pm i k_{j, z}(\xi) z} e^{i \xi x} d \xi
$$

Запишем теперь напряженность поля в рассматриваемых областях в следующем виде.
В области 1

$$
\begin{aligned}
\mathbf{E}_{1}(x, z)= & \frac{1}{2 \pi} \int_{-\infty}^{\infty}\left(\begin{array}{c}
1 \\
0 \\
-\frac{\xi}{k_{1, z}}
\end{array}\right) \hat{E}_{1, x}^{+}(\xi) e^{i k_{1, z} z} e^{i \xi x} d \xi \\
& +\frac{1}{2 \pi} \int_{-\infty}^{\infty}\left(\begin{array}{c}
1 \\
0 \\
\frac{\xi}{k_{1, z}}
\end{array}\right) \hat{E}_{1, x}^{-}(\xi) e^{-i k_{1, z} z} e^{i \xi x} d \xi,
\end{aligned}
$$

в области 2

$$
\begin{aligned}
\mathbf{E}_{2}(x, z)= & \frac{1}{2 \pi} \int_{-\infty}^{\infty}\left(\begin{array}{c}
1 \\
0 \\
-\frac{\xi}{k_{2, z}}
\end{array}\right) \hat{E}_{2, x}^{+}(\xi) e^{i k_{2, z} z} e^{i \xi x} d \xi \\
& +\frac{1}{2 \pi} \int_{-\infty}^{\infty}\left(\begin{array}{c}
1 \\
0 \\
\frac{\xi}{k_{2, z}}
\end{array}\right) \hat{E}_{2, x}^{-}(\xi) e^{-i k_{2, z} z} e^{i \xi x} d \xi,
\end{aligned}
$$

в области 3

$$
\mathbf{E}_{3}(x, z)=\frac{1}{2 \pi} \int_{-\infty}^{\infty}\left(\begin{array}{c}
1 \\
0 \\
-\frac{\xi}{k_{3, z}}
\end{array}\right) \hat{E}_{3, x}^{+}(\xi) e^{i k_{3, z}\left(z-d_{1}\right)} d \xi
$$

Полученные выражения для напряженности полей в рассматриваемых областях должны удовлетворять известным граничным условиям. Рассмотрим границу первой и второй областей $z=0$. На ней должны быть непрерывны тангенциальные компоненты напряженности электрического поля и нормальные компоненты электрической индукции, т. е.

$$
\begin{gathered}
E_{1, x}(x, 0)-E_{2}(x, 0)=0, \\
\varepsilon_{1} E_{1, z}(x, 0)-\varepsilon_{2} E_{2, z}(x, 0)=0 .
\end{gathered}
$$

где

$$
\begin{aligned}
& E_{1, x}(x, 0)=E_{1, x}^{+}(x, 0)+E_{1, x}^{-}(x, 0), \\
& E_{2, x}(x, 0)=E_{2, x}^{+}(x, 0)+E_{2, x}(x, 0), \\
& E_{1, z}(x, 0)=E_{1, z}^{+}(x, 0)+E_{1, z}(x, 0), \\
& E_{2, z}(x, 0)=E_{2, z}^{+}(x, 0)+E_{2, z}^{-}(x, 0) .
\end{aligned}
$$

Можно показать, что условие $\varepsilon_{j} E_{j, z}=$ const эквивалентно в нашем случае непрерывности тангенциальной компоненты магнитной индукции $B_{j, y} / \mu$ (в рассматриваемом случае у магнитного индукции существует только компонента вдоль оси $Y$ ). 
Из граничных условий (14) и (15), используя (11) и (12), при $z=0$ получим следующие два уравнения:

$$
\begin{gathered}
\hat{E}_{1, x}^{+}+\hat{E}_{1, x}^{-}-\hat{E}_{2, x}^{+}-\hat{E}_{2, x}^{-} e^{i k_{2, z} d_{1}}=0, \\
\varepsilon_{1}\left(-\frac{\xi}{k_{1, z}} \hat{E}_{1, x}^{+}+\frac{\xi}{k_{1, z} \hat{E}_{1, x}^{-}}\right) \\
-\varepsilon_{2}\left(-\frac{\xi}{k_{2, z}} \hat{E}_{2, x}^{+}+\frac{\xi}{k_{2, z}} \hat{E}_{2, x}^{-} e^{i k_{2, z} d_{1}}\right)=0,
\end{gathered}
$$

которые можно переписать в матричном виде:

$$
\begin{aligned}
& {\left[\begin{array}{cc}
1 & 1 \\
-\varepsilon_{1} \frac{\xi}{k_{1, z}} & \varepsilon_{1} \frac{\xi}{k_{1, z}}
\end{array}\right]\left(\begin{array}{c}
\hat{E}_{1, x}^{+} \\
\hat{E}_{1, x}^{-}
\end{array}\right)} \\
& =\left[\begin{array}{cc}
1 & e^{i k_{2, z} d_{1}} \\
-\varepsilon_{2} \frac{\xi}{k_{2, z}} & \varepsilon_{2} \frac{\xi}{k_{2, z}} e^{i k_{2, z} d_{1}}
\end{array}\right]\left(\begin{array}{c}
\hat{E}_{2, x}^{+} \\
\hat{E}_{2, x}^{-}
\end{array}\right)
\end{aligned}
$$

или

$$
\begin{aligned}
& \left(\begin{array}{c}
\hat{E}_{1, x}^{+} \\
\hat{E}_{1, x}^{-}
\end{array}\right)=\left[\begin{array}{cc}
1 & 1 \\
-\varepsilon_{1} \frac{\xi}{k_{1, z}} & \varepsilon_{1} \frac{\xi}{k_{1, z}}
\end{array}\right]^{-1} \\
& \times\left[\begin{array}{cc}
1 & e^{i k_{2, z} d_{1}} \\
-\varepsilon_{2} \frac{\xi}{k_{2, z}} & \varepsilon_{2} \frac{\xi}{k_{2, z}} e^{i k_{2, z} d_{1}}
\end{array}\right]\left(\begin{array}{c}
\hat{E}_{2, x}^{+} \\
\hat{E}_{2, x}^{-}
\end{array}\right)
\end{aligned}
$$

Аналогично рассмотрим границу второй и третьей областей $\left(z=d_{1}\right)$. Граничные условия запишутся в виде

$$
\begin{gathered}
E_{2, x}\left(x, d_{1}\right)-E_{3, x}\left(x, d_{1}\right)=0, \\
\varepsilon_{2} E_{2, z}\left(x, d_{1}\right)-\varepsilon_{1} E_{3, z}\left(x, d_{1}\right)=0,
\end{gathered}
$$

из которых следуют два матричных уравнения:

$$
\begin{gathered}
{\left[\begin{array}{cc}
e^{i k_{2, z} d_{1}} & 1 \\
-\varepsilon_{2} \frac{\xi}{k_{2, z}} e^{i k_{2, z} d_{1}} & \varepsilon_{2} \frac{\xi}{k_{2, z}} e^{i k_{2, z} d_{1}}
\end{array}\right]\left(\begin{array}{c}
\hat{E}_{2, x}^{+} \\
\hat{E}_{2, x}^{-}
\end{array}\right)} \\
=\left[\begin{array}{cc}
1 & 0 \\
-\varepsilon_{3} \frac{\xi}{k_{3, z}} & 0
\end{array}\right]\left(\begin{array}{c}
\hat{E}_{3, x}^{+} \\
0
\end{array}\right)
\end{gathered}
$$

и

$$
\begin{aligned}
\left(\begin{array}{c}
\hat{E}_{2, x}^{+} \\
\hat{E}_{2, x}^{-}
\end{array}\right)= & {\left[\begin{array}{cc}
e^{i k_{2, z} d_{1}} & 1 \\
-\varepsilon_{2} \frac{\xi}{k_{2, z}} e^{i k_{2, z} d_{1}} & \varepsilon_{2} \frac{\xi}{k_{2, z}}
\end{array}\right]^{-1} } \\
& \times\left[\begin{array}{cc}
1 & 0 \\
-\varepsilon_{3} \frac{\xi}{k_{3, z}} & 0
\end{array}\right]\left(\begin{array}{c}
\hat{E}_{3, x}^{+} \\
0
\end{array}\right) .
\end{aligned}
$$

Из матричных уравнений (16), (17) получаем

$$
\left(\begin{array}{c}
\hat{E}_{1, x}^{+} \\
\hat{E}_{1, x}^{-}
\end{array}\right)=M\left(\begin{array}{c}
\hat{E}_{3, x}^{+} \\
0
\end{array}\right)
$$

где матрица $M$ представляется в виде произведения трех матриц $\mathrm{M}=\mathrm{T}_{1} \mathrm{~T}_{2} \mathrm{~T}_{3}$, в котором

$$
\begin{gathered}
T_{1}=\left[\begin{array}{cc}
1 & 1 \\
-\varepsilon_{1} \frac{\xi}{k_{1, z}} & \varepsilon_{1} \frac{\xi}{k_{1, z}}
\end{array}\right]^{-1}=\frac{1}{2}\left(\begin{array}{cc}
1 & -\frac{k_{1, z}}{\varepsilon_{1} \xi} \\
1 & \frac{k_{1, z}}{\varepsilon_{1} \xi}
\end{array}\right), \\
T_{2}=\left(\begin{array}{cc}
1 & e^{i k_{2, z} d_{1}} \\
-\varepsilon_{2} \frac{\xi}{k_{2, z}} & \varepsilon_{2} \frac{\xi}{k_{2, z}} e^{i k_{2, z} d_{1}}
\end{array}\right)\left[\begin{array}{cc}
e^{i k_{2, z} d_{1}} & 1 \\
-\varepsilon_{2} \frac{\xi}{k_{2, z}} e^{i k_{2, z} d_{1}} & \varepsilon_{2} \frac{\xi}{k_{2, z}}
\end{array}\right] \\
=\left[\begin{array}{cc}
\cos \left(k_{2, z} d_{1}\right) & i \frac{k_{2, z}}{\varepsilon_{2} \xi} \sin \left(k_{2, z} d_{1}\right) \\
i \frac{\varepsilon_{2} \xi}{k_{2, z}} \sin \left(k_{2, z} d_{1}\right) & \cos \left(k_{2, z} d_{1}\right)
\end{array}\right], \\
T_{3}=\left[\begin{array}{cc}
1 & 0 \\
-\varepsilon_{3} \frac{\xi}{k_{3, z}} & 0
\end{array}\right] .
\end{gathered}
$$

Из (18) получаем $\hat{E}_{1, x}=M_{11} \hat{E}_{3, x}^{+}$и $\hat{E}_{1, x}^{-}=M_{21} \hat{E}_{3, x}^{+}$. Вводя функцию $R(\xi)=-M_{21} / M_{11}$, можно выразить отраженную волну как $\hat{E}_{1, x}^{-}=-R(\xi) \hat{E}_{1, x}^{+}$.

Отметим, что обобщение формулы (18) для многопленочной структуры из $N$ слоев можно произвести по индукции, которая приводит к следующим выражениям:

$$
\left(\begin{array}{c}
E_{1, x}^{+} \\
E_{1, x}^{-}
\end{array}\right)=\mathrm{M}\left(\begin{array}{c}
E_{N+2, x}^{+} \\
0
\end{array}\right),
$$

причем матрица М имеет вид

$$
\mathrm{M}=\mathrm{T}_{1}\left(\prod_{m=2}^{N+1} T_{m}\right) \mathrm{T}_{N+2},
$$

где

$$
\begin{gathered}
T_{1}=\frac{1}{2}\left[\begin{array}{cc}
1 & -\frac{k_{1, z}}{\varepsilon_{1} \xi} \\
1 & \frac{k_{1, z}}{\varepsilon_{1}}
\end{array}\right], \\
T_{m}=\left[\begin{array}{cc}
\cos \left(k_{m, z} d_{m-1}\right) & i \frac{k_{m, z}}{\varepsilon_{m} \xi} \sin \left(k_{m, z} d_{m-1}\right) \\
i \frac{\varepsilon_{m} \xi}{k_{m, z}} \sin \left(k_{m, z} d_{m-1}\right) & \cos \left(k_{m, z} d_{m-1}\right)
\end{array}\right], \\
T_{N+1}=\left[\begin{array}{cc}
1 & 0 \\
-\varepsilon_{N+2} \frac{\xi}{k_{N+2}, z} & 0
\end{array}\right] .
\end{gathered}
$$

В настоящей работе мы будем интересоваться падающей и отраженной волнами в первой среде. Напряженность поля в первой среде (11), учитывая вышесказанное, можно переписать в виде

$$
\begin{aligned}
& \mathbf{E}_{1}(x, z)=\frac{1}{2 \pi} \\
& \times \int_{-\infty}^{\infty}\left\{\left(\begin{array}{c}
1 \\
0 \\
-\frac{\xi}{k_{1, z}}
\end{array}\right) e^{i k_{1, z} z}-\left(\begin{array}{c}
1 \\
0 \\
\frac{\xi}{k_{1, z}}
\end{array}\right) R(\xi) e^{-i k_{1, z} z}\right\} \\
& \times \hat{E}_{1, x}(\xi) e^{i \xi x} d \xi
\end{aligned}
$$


Формула (19) показывает, что падающее поле можно разложить на плоские волны с разной гармонической вариацией по оси $x$ (т.е. $\left.e^{i \xi x}\right)$ и каждая такая плоская волна отразится от плоскослоистой структуры с коэффициентом отражения $R(\xi)$ под углом, соответствующим данному $\xi$. Чтобы доказать это, рассмотрим сначала падение на пленку плоской неограниченной волны единичной амплитуды. Падающее поле на плоской границе возбуждает поле вариацией по оси $x$ в виде $e^{i k_{1 x} x}=e^{i k_{1} \sin \alpha x}$, где $k_{1}=n_{1} \omega / c-$ волновое число в первой среде, $c$ - скорость света в вакууме, $n_{1}=\sqrt{\varepsilon_{1}}-$ показатель преломления в первой среде.

Пусть падающая волна поляризована в плоскости падения. Тогда электрическое поле падающей волны представляется (рис. 2) в виде

$$
\begin{aligned}
\mathbf{E}_{1}^{\text {in }}(x, z) & =\mathbf{E}_{1}^{+}(x, z) \\
& =e^{i\left(k_{1} \sin \alpha+k_{1} z \cos \alpha\right)}\left(\cos \alpha \mathbf{e}_{x}-\sin \alpha \mathbf{e}_{z}\right) .
\end{aligned}
$$

При $z=0$ получим

$$
\begin{aligned}
\hat{E}_{1, x}(\xi) & =\int_{-\infty}^{\infty} E_{x}^{+}(x) e^{-i \xi x} d x=\int_{-\infty}^{\infty} \cos \alpha e^{i k_{1} \sin \alpha} e^{-i \xi x} d x \\
& =2 \pi \cos \alpha \delta\left(k_{1} \sin \alpha-\xi\right) .
\end{aligned}
$$

Из формулы (19) получаем (с учетом, что $\left.\left.k_{1, z}\right|_{\xi=k_{1} \sin \alpha}=k_{1} \sin \alpha\right)$

$$
\begin{aligned}
\mathbf{E}_{1}(x, z) & =\frac{1}{2 \pi} \\
& \times \int_{-\infty}^{\infty}\left\{\left(\begin{array}{c}
1 \\
0 \\
-\frac{\xi}{k_{1, z}}
\end{array}\right) e^{i k_{1, z} z}-\left(\begin{array}{c}
1 \\
0 \\
\frac{\xi}{k_{1, z}}
\end{array}\right) R(\xi) e^{-i k_{1, z} z}\right\} \\
& \times 2 \pi \cos \alpha \delta\left(k_{1} \sin \alpha-\xi\right) e^{i \xi x} d \xi \\
& =\left(\begin{array}{c}
\cos \alpha \\
0 \\
-\sin \alpha
\end{array}\right) e^{i\left(k_{1} x \sin \alpha+k_{1} z \cos \alpha\right)} \\
& -R\left(k_{1} \sin \alpha\right)\left(\begin{array}{c}
\cos \alpha \\
0 \\
\sin \alpha
\end{array}\right) e^{i\left(k_{1} x \sin \alpha-k_{1} z \cos \alpha\right)} .
\end{aligned}
$$

Это означает, что полное поле есть поле падающей и отраженной плоских волн, причем коэффициент отражения равен $R=R\left(k_{1} \sin \alpha\right)$.

Пусть теперь на пленку падает ограниченная плоская волна, причем при $z=0$ волна (рис. 2) падает только на отрезок $x \in[-L / 2, L / 2]$. Тогда

$$
\begin{aligned}
\hat{E}_{1, x}^{\mathrm{in}}(\xi) & =\int_{-\infty}^{\infty} E_{x}^{+}(x) e^{-i \xi x} d x=\int_{-L / 2}^{L / 2} E_{x}^{+}(x) e^{-i \xi x} d x \\
& =\int_{-L / 2}^{L / 2} \cos \alpha e^{i k_{1} x \sin \alpha} e^{-i \xi x} d x \\
& =2 \cos \alpha \frac{\sin \left(\left(k_{1} \sin \alpha-\xi\right) L / 2\right)}{\left(k_{1} \sin \alpha-\xi\right)} .
\end{aligned}
$$

Подставляя полученное выражение в общую формулу (19), получим

$$
\begin{aligned}
& \mathbf{E}_{1}(x, z)=\frac{L \cos \alpha}{2 \pi} \int_{-\infty}^{\infty}\left(\begin{array}{c}
1 \\
0 \\
-\frac{\xi}{k_{1, z}}
\end{array}\right) \\
& \times \frac{\sin \left(\left(k_{1} \sin \alpha-\xi\right) L / 2\right)}{\left(k_{1} \sin \alpha-\xi\right) L / 2} e^{i\left(\xi x+k_{1,2} z\right)} d \xi \\
& -\frac{L \cos \alpha}{2 \pi} \int_{-\infty}^{\infty}\left(\begin{array}{c}
1 \\
0 \\
\frac{\xi}{k_{1, z}}
\end{array}\right) \\
& \times \frac{R(\xi) \sin \left(\left(k_{1} \sin \alpha-\xi\right) L / 2\right)}{\left(k_{1} \sin \alpha-\xi\right) L / 2} e^{i\left(\xi x-k_{1, z} z\right)} d \xi
\end{aligned}
$$

В результате получено выражение для полного поля. Первый интеграл справа в (20) представляет собой сумму плоских волн, на которые можно разложить падающую волну с ограниченной апертурой. Второй интеграл есть соответствующая сумма отраженных плоских волн. Влияние апертуры на отраженную волну приводит к разделению энергии падающей волны по волнам в различных направлениях в окрестности угла $\alpha$.

Итак, выражение для напряженности отраженного поля можно записать в виде

$$
\begin{aligned}
& \mathbf{E}_{1}^{r}(x, z)=-\frac{L \cos \alpha}{2 \pi} \int_{-\infty}^{\infty}\left(\begin{array}{c}
1 \\
0 \\
\frac{\xi}{k_{1, z}}
\end{array}\right) \\
& \quad \times \frac{\sin \left(\left(k_{1} \sin \alpha-\xi\right) L / 2\right)}{\left(k_{1} \sin \alpha-\xi\right) L / 2} R(\xi) e^{i\left(\xi x-k_{1, z} z\right)} d \xi .
\end{aligned}
$$

Тогда амплитуда поля, отраженного под углом $\beta$ в пределах малого угла $d \beta$ (с учетом, что $\xi=k_{1} \sin \beta$, $\left.d \xi=k_{1} \cos \beta d \beta\right)$, равна

$$
\begin{aligned}
\frac{d E_{1, a}^{r}}{d \beta}= & \frac{k_{1} L \cos \alpha}{2 \pi} \\
& \times\left|\frac{\sin \left((\sin \alpha-\sin \beta) k_{1} L / 2\right)}{(\sin \alpha-\sin \beta) k_{1} L / 2} R\left(k_{1} \sin \beta\right)\right| .
\end{aligned}
$$


Таблица 1.

\begin{tabular}{c|c|c|c}
\hline $\begin{array}{c}\text { Порядковый } \\
\text { номер среды, } j\end{array}$ & $\begin{array}{c}\text { Название } \\
\text { слоя }\end{array}$ & $\begin{array}{c}\text { Относительная диэлектрическая } \\
\text { проницаемость }\end{array}$ & $\begin{array}{c}\text { Толщина } \\
\text { пленки, nm }\end{array}$ \\
\hline 1 & Призма & $1.6^{2}$ & $\begin{array}{c}\text { Полубесконечная } \\
\text { среда }\end{array}$ \\
\hline 2 & $\begin{array}{c}\text { Серебряная } \\
\text { пленка }\end{array}$ & $\varepsilon_{m}=-18.2+i 0.5$ & 53.8 \\
\hline 3 & $\begin{array}{c}\text { Свободное пространство } \\
\text { (воздух) }\end{array}$ & 1 & $\begin{array}{c}\text { Полубесконечная } \\
\text { среда }\end{array}$
\end{tabular}

Таблица 2.

\begin{tabular}{c|c|c|c}
\hline $\begin{array}{c}\text { Порядковый } \\
\text { номер среды, } j\end{array}$ & $\begin{array}{c}\text { Название } \\
\text { слоя }\end{array}$ & $\begin{array}{c}\text { Относительная диэлектрическая } \\
\text { проницаемость }\end{array}$ & $\begin{array}{c}\text { Толщина } \\
\text { пленки, пm }\end{array}$ \\
\hline 1 & Призма & $\begin{array}{c}\text { Полубесконечная } \\
\text { среда }\end{array}$ \\
\hline 2 & Согласующий слой & $\varepsilon_{2}=1.2^{2}$ \\
\hline 3 & Прозрачный электрод & $\varepsilon_{3}=1.8^{2}+i 0.002$ \\
\hline 4 & Оптически плотная пленка & $\varepsilon_{4}=2.2^{2}$ \\
\hline 5 & Прозрачный электрод & $\varepsilon_{5}=1.8^{2}+i 0.002$ \\
\hline 6 & Свободное & $\varepsilon_{6}=1$ \\
\hline
\end{tabular}

Полученное распределение дает нам угловое распределение амплитуды отраженного поля (при условии падения волны единичной амплитуды с апертурой $D=L \cos \alpha)$.

\section{Влияние конечного размера датчиков поверхностной волны на их чувствительность}

Рассмотрим теперь, как влияет конечный размер апертуры датчиков на поверхностных волнах на их чувствительность. Начнем с датчика на поверхностных плазмонах, распространяющихся по поверхности серебряной пленки (рис. 1). Как было показано в [22], в случае бесконечной апертуры падающей волны существует оптимальная толщина серебряной пленки. Для призмы с показателем преломления $n_{1}=1.6$ оптимальные величины приведены в табл. 1. Длина падающей волны в вакууме $\lambda=633 \mathrm{~nm}$. В этом случае волна бесконечно большой апертуры, падающая под углом $\alpha$, порождает отраженную волну строго под углом $\alpha$ (распределение по углам отражения является $\delta$-функцией). Зависимость коэффициента отражения от угла падения $\alpha$ для этого случая показана на рис. 3 .

Если теперь осветить пленку пучком ограниченной апертуры под оптимальным углом $\alpha_{\text {opt }}=40.041^{\circ}$, то изза конечности $L$ распределение отраженного поля по углам отражения $\beta$ не будет $\delta$-функцией. На рис. 4 показана зависимость функции

$$
\begin{aligned}
F(\beta) & =\frac{2 \pi}{k_{1} L \cos \alpha_{\mathrm{opt}}} \frac{d E_{1, a}^{r}}{d \beta} \\
& =\left|\frac{\sin \left(\left(\sin \alpha_{\mathrm{opt}}-\sin \beta\right) k_{1} L / 2\right)}{\left(\sin \alpha_{\mathrm{opt}}-\sin \beta\right) k_{1} L / 2} R\left(k_{1} \sin \beta\right)\right| .
\end{aligned}
$$

Функция $F(\beta)$ пропорциональна амплитуде напряженности электрического поля волны, отраженной под углом $\beta$, и определяет угловую зависимость отражения. На рис. 4 зависимость $F(\beta)$ показана для $L=30,20,10$, $1 \mu \mathrm{m}$ (кривые $1,2,3,4$ соответственно).

Из рис. 4 видно, что с увеличением $L$ и, значит, апертуры падающей волны $D=L \cos \alpha$ угловой разброс пространственных гармоник уменьшается. Угловой разброс с точностью до константы можно оценить функцией $\Delta \beta \sim \lambda_{1} / D$, которая точно определяется выражением (22). Рассматривая зависимости рис. 4 с точки зрения чувствительности датчиков, можно сделать следующий вывод. Если датчик анализирует угловой спектр отраженных волн как в [23], то при дифракционной ширине $\Delta \beta$ в несколько раз большей угловой ширины минимума, связанного с поверхностной волной (рис. 3), дифракционное уширение углового спектра, связанное с конечностью апертуры падающей волны, не будет влиять на чувствительность датчика. Размер области, на 


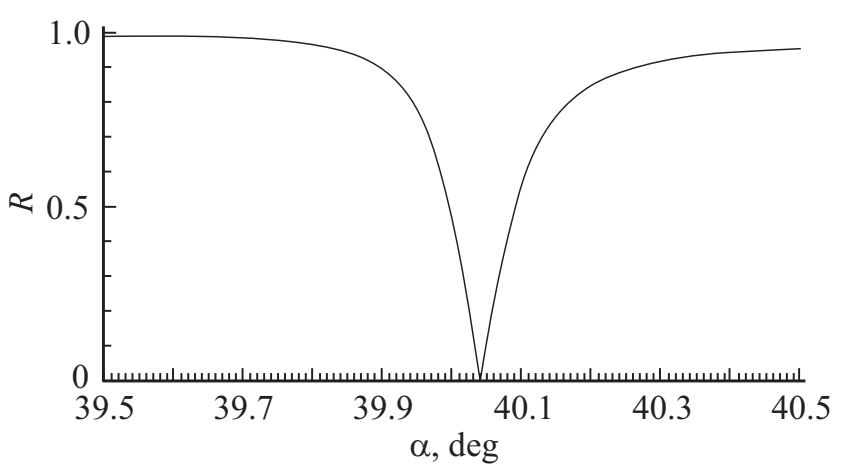

Рис. 3. Коэффициент отражения при падении бесконечной плоской волны р-поляризации на серебряную пленку (табл. 1) как функция угла падения $\alpha$.

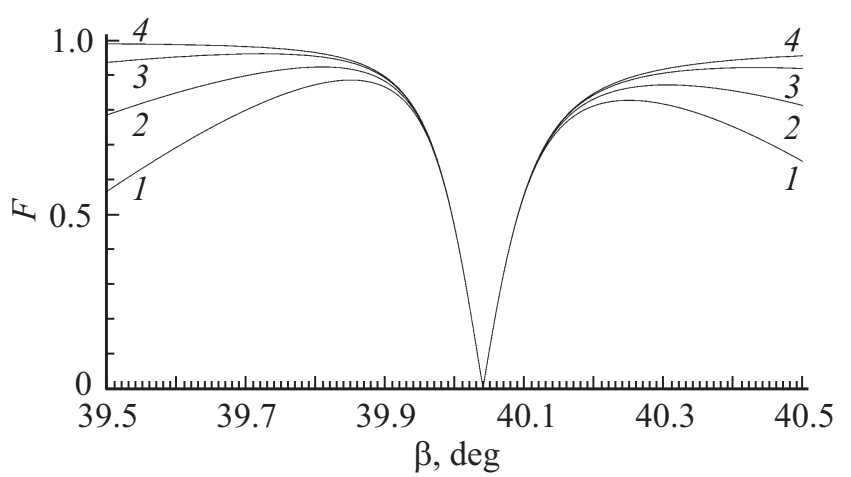

Рис. 4. Распределение амплитуды отраженного сигнала $F(\beta)$ при падении плоской $p$-волны ограниченной апертуры на серебряную пленку (табл. 1) по углу отражения $\beta L=30$ (1), 20 (2), 10 (3), $1 \mu \mathrm{m}(4)$.

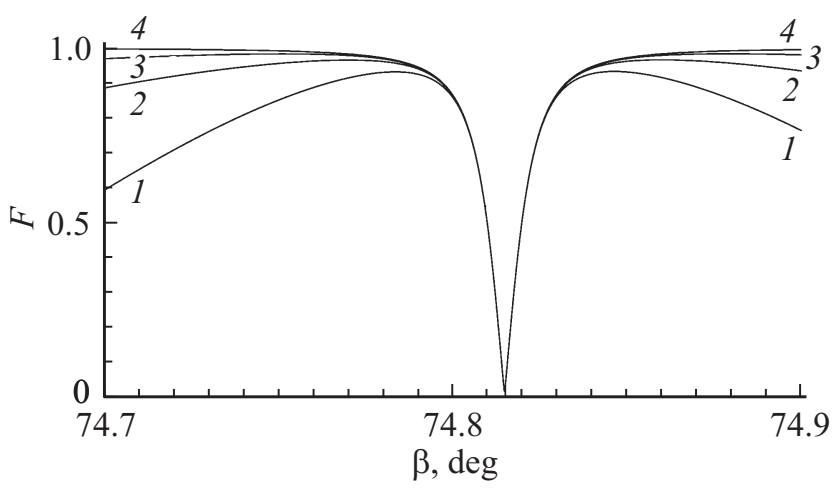

Рис. 5. Распределение амплитуды отраженного сигнала $F(\beta)$ при падении плоской $p$-волны ограниченной апертуры на многопленочную структуру (табл. 2) по углу отражения $\beta$. $L=400$ (1), 200 (2), 100 (3), $20 \mu \mathrm{m}(4)$.

которую падает волна, должна быть порядка $50 \mu \mathrm{m}$ и меньше.

Полученные результаты можно проиллюстрировать еще одним примером. Как было показано в работе [20], сенсоры по схеме Кречмана можно построить на поверхностных волнах в многопленочных структурах. При этом чувствительность сенсора, определяемая поглощением поверхностной волны, можно независимо контролировать толщиной пленки проводящего материала. В работе [20] было показано, что таким образом можно увеличить чувствительность сенсора в десятки раз по сравнению с сенсорами на поверхностных плазмонах. В этой работе были, в частности, найдены параметры пленок оптимальной многопленочной структуры (табл. 2).

На рис. 5 показаны для этой многопленочной структуры расчеты углового распределения отраженной волны по формуле (22). На рис. 5 зависимость $F(\beta)$ показана для $=400,200,100,20 \mu \mathrm{m}$ (кривые $1,2,3,4$ соответственно).

Как и в первом примере структуры на поверхностных плазмонах, для второго примера дифракционное уширение углового спектра, связанное с конечностью апертуры падающей волны, не будет влиять на чувствительность датчика, если размер области, на которую падает волна, порядка $400 \mu \mathrm{m}$ и меньше.

\section{Заключение}

Предложен теоретический метод исследования дифракционных явлений при отражении плоской электромагнитной волны ограниченной апертуры от плоскослоистой многопленочной структуры с однородными пленками. Показано, что ограниченный размер апертуры падающей волны не является принципиальным ограничением чувствительности сенсоров на поверхностных волнах. Полученные результаты могут быть применены для создания многоканальных сенсоров поверхностных волн, обладающих рекордной чувствительностью, в современных оптических многоканальных биологических, химических и физических сенсорных системах.

\section{Список литературы}

[1] Homola J., Yee S.S., Gauglitz G. // Sens. Actuators B. 1999. V. 54. P. 3.

[2] Barnes W.L., Dereux A., Ebbesen T.W. // Nature. 2003. V. 424. P. 824.

[3] Homola J. // Chem. Rev. 2008. V. 108. P. 462.

[4] Spoto G., Minunni M. // J. Phys. Chem. Lett. 2012. V. 3. P. 2682.

[5] Raether H. Surface Plasmons. Berlin: Springer-Verlag, 1988. $353 \mathrm{p}$.

[6] Barnes W.L. // J. Opt. A: Pure Appl. Opt. 2006. V. 8. P. S87.

[7] Kretschmann E., Raether H.Z. // Naturforsch. 1968. V. A 23. P. 2135.

[8] Piliarik M., Homola J. // Opt. Express. 2009. V. 17. N 19. P. 16505.

[9] Liedberg B., Nylander C., Lundstrom I. // Sens. Actuators. 1983. V. 4. P. 299.

[10] Liedberg B., Nylander C., Lundstrom I. // Biosens. Bioelectron. 1995. V. 10. P. i-ix.

[11] Garabedian R., Gonzalez C., Richards J. et al. // Sens. Actuators A. 1994. V. 43. P. 202. 
[12] Yeatman E.M. // Biosens. Bioelectron. 1996. V. 11. P. 635.

[13] Sarid D. // Phys. Rev. Lett. 1981. V. 47. P. 1927.

[14] Matsubara K., Kawata S., Minami S. // Opt. Lett. 1990. V. 15. P. 75.

[15] Yang F., Bradberry G.W., Sambles J.R. // Phys. Rev. Lett. 1991. V. 66. P. 2030.

[16] Kessler M.A., Hall E.A.H. // Thin Solid Films. 1996. V. 272. P. 161.

[17] Nenninger G.G., Tobiska P., Homola J., Yee S.S. // Sens. Actuators B. 2001. V. 74. P. 145.

[18] Toyama S., Doumae N., Shoji A., Ikariyama Y. // Sens. Actuators B. 2000. V. 65. P. 32.

[19] Airoudj A., Debarnot D., Beche B., Poncin-Epaillard F. // Anal. Chem. 2008. V. 80. P. 9188.

[20] Петрин А.Б., Вольпян О.Д., Сигов А.С. // Опт. и спектр. 2017. T. 123. № 5. C. 786.

[21] Новотный Л., Хехт Б. Основы нанооптики. Пер. с англ. под ред. В.В. Самарцева. М.: Физматлит, 2009. 484 с.

[22] Петрин А.Б., Вольпян О.Д., Сигов А.С. // ЖТФ. 2018. Т. 88. № 3. C. 433.

[23] Löfas S., Malmqvist M., Rönnberg, et al. // Sens. Actuators B. 1991. V. 5. P. 79. 